\title{
ON P.P.-RINGS WHICH ARE REDUCED
}

\author{
XIAOJIANG GUO AND K. P. SHUM
}

Received 16 March 2006; Accepted 19 March 2006

Denote the $2 \times 2$ upper triangular matrix rings over $\mathbb{Z}$ and $\mathbb{Z}_{p}$ by $\operatorname{UTM}_{2}(\mathbb{Z})$ and $\operatorname{UTM}_{2}\left(\mathbb{Z}_{p}\right)$, respectively. We prove that if a ring $R$ is a p.p.-ring, then $R$ is reduced if and only if $R$ does not contain any subrings isomorphic to $\mathrm{UTM}_{2}(\mathbb{Z})$ or $\mathrm{UTM}_{2}\left(\mathbb{Z}_{p}\right)$. Other conditions for a p.p.-ring to be reduced are also given. Our results strengthen and extend the results of Fraser and Nicholson on r.p.p.-rings.

Copyright (c) 2006 Hindawi Publishing Corporation. All rights reserved.

\section{Introduction}

Throughout the paper, all rings are associative rings with identity 1 . The set of all idempotents of a ring $R$ is denoted by $E(R)$. Also, for a subset $X \subseteq R$, we denote the right [resp., left] annihilator of $X$ by $r(X)$ [resp., $\ell(X)]$.

We call a ring $R$ a left p.p.-ring [3], in brevity, an l.p.p.-ring, if every principal left ideal of $R$, regarded as a left $R$-module, is projective. Dually, we may define the right p.p.-rings (r.p.p.-rings). We call a ring $R$ a p.p.-ring if $R$ is both an l.p.p.- and r.p.p.-ring. It can be easily observed that the class of p.p.-rings contains the classes of regular (von Neumann) rings, hereditary rings, Baer rings, and semihereditary rings as its proper subclasses. In the literature, p.p.-rings have been extensively studied by many authors and many interesting results have been obtained (see [1-7]). It is noteworthy that the definition of p.p.-rings can also be extended to semigroups.

We now call a ring $R$ reduced if it contains no nonzero nilpotent elements. Obviously, the left annihilator $\ell(X)$ of $X$ in a reduced ring $R$ is always a two-sided ideal of $R$. Moreover, if $R$ is a reduced ring, then $e f=0$ if and only if $f e=0$ for any nonzero idempotents $e, f \in R$. Reduced rings with the maximum condition on annihilator were first studied by Cornish and Stewart [2]. By using the concept of annihilator and reduced ring, Fraser and Nicholson [3] showed that a ring $R$ is a reduced p.p.-ring if and only if $R$ is a (left, right) p.p.-ring in which every idempotent is central.

In this paper, we will prove that a p.p.-ring $R$ is reduced if and only if $R$ contains no subrings which are isomorphic to the matrix rings $\mathrm{UTM}_{2}(\mathbb{Z})$ or $\mathrm{UTM}_{2}\left(\mathbb{Z}_{p}\right)$. Thus, our 
results strengthen and extend the results obtained by Fraser and Nicholson in [3]. Also, some of our results can be applied to r.p.p.-monoids with zero.

\section{Definitions and basic results}

The following crucial lemma of p.p.-rings was given by Fraser and Nicholson in [3].

Lemma 2.1 [3]. Let $R$ be a ring and $a \in R$. Then $R$ is an l.p.p.-ring if and only if $\ell(a)=R e$ for some idempotent $e \in E(R)$.

By using Lemma 2.1, we can give some properties of a p.p.-ring which is reduced.

Theorem 2.2. Let $R$ be a p.p.-ring and $E(R)$ the set of all idempotents of $R$. Then the following statements are equivalent:

(i) $R$ is reduced;

(ii) ef $=$ fe for all e, $f \in E(R)$;

(iii) $E(R)$ is a subsemigroup of the semigroup $(R, \cdot)$;

(iv) $e f=0$ if and only if $f e=0$ for all $e, f \in E(R)$;

(v) $e R=R e$ for all $e \in E(R)$.

Proof. (i) $\Rightarrow$ (ii) $\Rightarrow$ (iii) are trivial.

(iii) $\Rightarrow$ (iv). Let $e, f \in E(R)$. Suppose that $e f=0$. Then by (iii), we have $f e \in E(R)$ and so $f e=(f e)^{2}=f(e f) e=0$. Similarly, we can show that if $f e=0$, then $e f=0$. This proves (iv).

(iv) $\Rightarrow(\mathrm{v})$. Let $x \in r(e)$. Then $e x=0$ and so $e \in \ell(x)$. Since $R$ is a p.p.-ring, by Lemma 2.1, we have $\ell(x)=R f$, for some $f \in E(R)$. Now, by Pierce decomposition, we have $R=$ $R(1-f) \oplus R f$ and hence $\ell(1-f)=R f$. Consequently $e \in \ell(1-f)=\ell(x)$ and thereby $e(1-f)=0$ since $e x=0$. Because $(1-f) \in E(R)$, by (iv), we have $(1-f) e=0$. It is now easy to check that $e+x e \in E(R)$. Since $(e+x e)(1-f)=0$, we have, by (iv), $0=(1-$ $f)(e+x e)=(1-f) x e$. However, by $\ell(x)=R f$ and $1 \in R$, we have $f x=0$ so that $f x e=0$. This leads to $x e=(1-f) x e+f x e=0$, and thereby $x \in \ell(e)$. Thus $r(e) \subseteq \ell(e)$. Dually, we can show that $\ell(e) \subseteq r(e)$. Therefore $r(e)=\ell(e)$. Thus, for all $e \in R, r(1-e)=\ell(1-e)$, that is, $e R=R e$. This proves $(\mathrm{v})$. [3].

$(v) \Rightarrow(i)$. Since (v) easily yields that the idempotents of $R$ are central, so (v) $\Rightarrow(i)$ by

The following example illustrates that there exists a p.p.-ring which is not reduced.

Example 2.3. Let $\mathrm{UTM}_{2}(\mathbb{R})$ be the subring of the matrix ring $M_{2}(\mathbb{R})$ consisting of all $2 \times 2$ upper triangular matrices over the field $\mathbb{R}$. We claim that $\mathrm{UTM}_{2}(\mathbb{R})$ is a p.p.-ring. In order to establish our claim, let

$$
A=\left(\begin{array}{ll}
a & c \\
0 & b
\end{array}\right), \quad B=\left(\begin{array}{ll}
x & z \\
0 & y
\end{array}\right)
$$

be elements of $\mathrm{UTM}_{2}(\mathbb{R})$. Then we see immediately that $A B=\left(\begin{array}{ll}0 & 0 \\ 0 & 0\end{array}\right)$ if and only if $a x=0$, $b y=0$ and $a z+c y=0$. The following cases now arise. 
(i) $x \neq 0$ and $y \neq 0$. In this case, we have $A B=\left(\begin{array}{ll}0 & 0 \\ 0 & 0\end{array}\right)$ if and only if $a=b=c=0$. Hence, we have

$$
\ell(B)=\left\{\left(\begin{array}{ll}
0 & 0 \\
0 & 0
\end{array}\right)\right\}=\mathrm{UTM}_{2}(\mathbb{R})\left(\begin{array}{ll}
0 & 0 \\
0 & 0
\end{array}\right) .
$$

(ii) $x \neq 0$ and $y=0$. In this case, we have $A B=\left(\begin{array}{ll}0 & 0 \\ 0 & 0\end{array}\right)$ if and only if $a=0$. This leads to

$$
\ell(B)=\left\{\left(\begin{array}{ll}
0 & c \\
0 & b
\end{array}\right): b, c \in \mathbb{R}\right\}=\mathrm{UTM}_{2}(\mathbb{R})\left(\begin{array}{ll}
0 & 0 \\
0 & 1
\end{array}\right) .
$$

(iii) $x=0$ and $y \neq 0$. In this case, we have $A B=\left(\begin{array}{ll}0 & 0 \\ 0 & 0\end{array}\right)$ if and only if $b=0$ and $c=a z y^{-1}$. This leads to

$$
\ell(B)=\left\{\left(\begin{array}{cc}
a & a z y^{-1} \\
0 & 0
\end{array}\right): a \in R\right\}=\operatorname{UTM}_{2}(\mathbb{R})\left(\begin{array}{cc}
1 & z y^{-1} \\
0 & 0
\end{array}\right) .
$$

Summing up the above cases, we can easily see that $\ell(B)$ of $\mathrm{UTM}_{2}(\mathbb{R})$ is generated by an idempotent. Clearly, $\mathrm{UTM}_{2}(\mathbb{R})$ is not reduced.

\section{Main theorem}

In proving the main theorem of this paper, we first denote by $o(r)$ the (additive) order of $r \in R$, that is, the smallest positive integer $n$ such that $n r=0$. If $r$ is of infinite order, then we simply write $o(r)=\infty$.

We now prove a useful lemma for p.p.-rings.

Lemma 3.1. Let $R$ be a p.p.-ring with 1 such that $e f=0$ but $f e \neq 0$ for some $e, f \in E(R)$. Then, $o(e)=o(f)=o(f e)$, and if $o(e)<\infty$, then there exist $u, v \in E(R)$ and a prime $p$ such that $o(u)=o(v)=o(v u)=p$ with $u v=0$ but $v u \neq 0$.

Proof. Since $R$ is a p.p.-ring, by Theorem $2.2, R$ is clearly not reduced. Also, since $1 \in$ $R$, by Lemma 2.1, there exists some $g, h \in E(R)$ such that $\ell(f e)=R(1-g)$ and $r(f e)=$ $(1-h) R$. These lead to $\ell(f e)=\ell(g)$ and $r(f e)=r(h)$. Since $1-f \in \ell(f e)$, we have $(1-$ $f) g=0$ and so $g=f g$. Since $g=f g$, we see that $g f \in E(R)$ and $\ell(g)=\ell(g f)$. Thus, $(1-g f) f e=0$ since $(1-g f) g=0$ and $\ell(g)=\ell(f e)$, that is, $f e-g f e=0$. Thereby, we have $g f e=f e$. Similarly, we can prove that there exists $h \in E(R)$ such that $h=h e, e h \in$ $E(R), r(e h)=r(f e)$, and $f e=f e h$. Hence, $f e=g f e h=(g f)(e h)$. On the other hand, we have $(e h)(g f)=e(h e)(f g) f=0$. Because $\ell(f e)=\ell(g f)$ and $r(f e)=r(e h)$, we can easily see that $o(g f)=o(e h)=o(f e)$.

Now two cases arise.

(i) $o(g f)=\infty$. In this case, there is nothing to prove.

(ii) $o(g f)<\infty$. Without loss of generality, let $o(g f)=p k$, where $p$ is a prime number. Then, we can easily check that $o(k f e)=p$. By using similar arguments as above, we also have $u, v \in E(R)$ such that $o(u)=o(v)=o(k f e)$ with $u v=0$ but $v u \neq 0$. Hence, $u$ and $v$ are the required idempotents in $R$. The proof is completed. 
We now formulate the following main theorem.

Theorem 3.2. Let $R$ be a p.p.-ring. Then $R$ is reduced if and only if $R$ has no subrings which are isomorphic either to $\mathrm{UTM}_{2}(\mathbb{Z})$ or to $\mathrm{UTM}_{2}\left(\mathbb{Z}_{p}\right)$, where $p$ is a prime.

Proof. The necessity part of the theorem follows from Theorem 2.2 since $\mathrm{UTM}_{2}(\mathbb{Z})$ and $\mathrm{UTM}_{2}\left(\mathbb{Z}_{p}\right)$ both contain some noncommutating idempotents.

To prove the sufficiency part of the theorem, we suppose that $R$ is not reduced. Then we can let $i, j \in E(R)$ such that $i j=0, j i \neq 0$, and $o(i)=o(j)=o(j i)$; and $o(i)=o(j)=$ $o(j i)=p$ if $o(i)<\infty$, where $p$ is a prime. Consider the subring of $R$ generated by $i$ and $j$. Clearly, $\{0, i, j, j i\}$ forms a subsemigroup of $R$ under ring multiplication and so $S=\{a i+$ $b j i+c i: a, b, c \in \mathbb{Z}\}$ forms a subring of $R$, under the ring multiplication and addition.

Now, we define a mapping $\theta: \mathrm{UTM}_{2}(\mathbb{Z}) \rightarrow S$ by

$$
\left(\begin{array}{ll}
a & b \\
0 & c
\end{array}\right) \longmapsto a j+(b-c) j i+c i
$$

Then, we can easily verify that $\theta$ is a surjective homomorphism of $\mathrm{UTM}_{2}(\mathbb{Z})$ onto $S$.

We now consider the kernel of $\theta$. Suppose that $A=\left(\begin{array}{cc}a & b \\ 0 & c\end{array}\right) \in \operatorname{ker} \theta$. Then we have $a j+$ $(b-c) j i+c i=0$. Multiplying $i$ on the left gives $c i=0$, and multiplying $j$ on the right gives $a j=0$. Hence, we have $(b-c) j i=0$.

The following cases arise.

(i) $o(i)=o(j)=o(j i)=\infty$. Then $a=0, c=0$, and $(b-c)=0$. Thus $a=b=c=0$ and thereby $A=0$. Hence $\operatorname{ker} \theta=\{0\}$ and $\theta$ is an isomorphism.

(ii) $o(i)=o(j)=o(j i)=p$. In this case, we have $p|a, p| c$, and $p \mid(b-c)$. Hence $p \mid$ $a, p \mid c$, and $p \mid b$. Consequently $\operatorname{ker} \theta=\left\{\left(\begin{array}{ll}a & b \\ 0 & c\end{array}\right): p|a, p| b\right.$, and $\left.p \mid c\right\}$. Observing that $\mathrm{UTM}_{2}(Z) / \operatorname{ker} \theta \cong \operatorname{UTM}_{2}\left(Z_{p}\right)$, we have $S \cong \operatorname{UTM}_{2}\left(Z_{p}\right)$. This contradicts our assumption and therefore our proof is completed.

As an application of our main theorem, we give a new criterion for a p.p.-ring to be reduced.

Theorem 3.3. Let $R$ be a p.p.-ring having no subrings isomorphic to $\mathrm{UTM}_{2}\left(Z_{p}\right)$ for prime $p$. If $o(e)<\infty$ for all $e \in E(R)$, then $R$ is reduced.

In fact, Theorem 3.3 follows from the following lemma.

Lemma 3.4. Let $R$ be a p.p.-ring having no subring isomorphic to $\mathrm{UTM}_{2}\left(\mathbb{Z}_{p}\right)$. Suppose that at least one of the idempotents $e, f \in E(R)$ has a prime order $p$. Then $e f=0$ if and only if $f e=0$.

Proof. Suppose that $e f=0$ but $f e \neq 0$. Also, suppose that $e$ or $f$ has a prime order $p$. Then, $f e$ must have an order $p$. Now, by using the arguments in the proof of Lemma 3.1, we can construct some idempotents $g, h \in R$ and that $o(g)=o(h)=o(h g)=p$ such that $h g=f e$ but $g h=0$. By using the arguments in the proof of Theorem 3.2, we can show similarly that the subring $S=\langle g, h\rangle$ of the ring $R$ (the subring of $R$ generated by $f$ and $g$ ) is isomorphic to $\operatorname{UTM}_{2}\left(\mathbb{Z}_{p}\right)$. However, this is clearly a contradiction. Thus, we have $f e=0$. This proves Lemma 3.4. 


\section{Acknowledgments}

The authors would like to thank the referee for giving them some useful suggestions which help to modify the presentation of the paper. This work is jointly supported by the Natural Science Foundation of Jiangxi Province, the Science Foundation of the Education Department of Jiangxi Province, and the Foundation of Jiangxi Normal University, China. The research is partially supported by a UGC (HK) Grant 2160210 (04/05).

\section{References}

[1] E. P. Armendariz, A note on extensions of Baer and P.P.-rings, Australian Mathematical Society 18 (1974), 470-473.

[2] W. H. Cornish and P. N. Stewart, Rings with no nilpotent elements and with the maximum condition on annihilators, Canadian Mathematical Bulletin 17 (1974), 35-38.

[3] J. A. Fraser and W. K. Nicholson, Reduced p.p.-rings, Mathematica Japonica 34 (1989), no. 5, $715-725$.

[4] Y. Hirano, M. Hongan, and M. Ôhori, On right p.p.-rings, Mathematical Journal of Okayama University 24 (1982), no. 2, 99-109.

[5] C. Y. Hong, N. K. Kim, and T. K. Kwak, Ore extensions of Baer and p.p.-rings, Journal of Pure and Applied Algebra 151 (2000), no. 3, 215-226.

[6] S. Jøndrup, p.p.-rings and finitely generated flat ideals, Proceedings of the American Mathematical Society 28 (1971), 431-435.

[7] Z. Liu and J. Aksan, p.p.-rings of generalized power series, Acta Mathematica Sinica. English Series 16 (2000), no. 4, 573-578.

Xiaojiang Guo: Department of Mathematics, Jiangxi Normal University, Nanchang,

Jiangxi 330027, China

E-mail address: xjguo@jxnu.edu.cn

K. P. Shum: Faculty of Science, The Chinese University of Hong Kong, Shatin, Hong Kong

E-mail address: kpshum@math.cuhk.edu.hk 


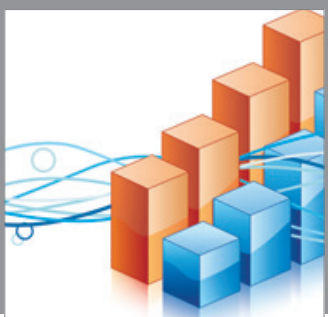

Advances in

Operations Research

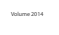

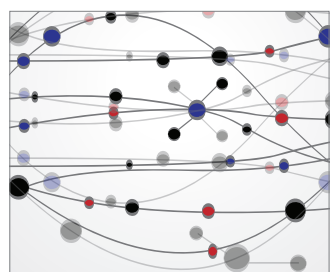

\section{The Scientific} World Journal
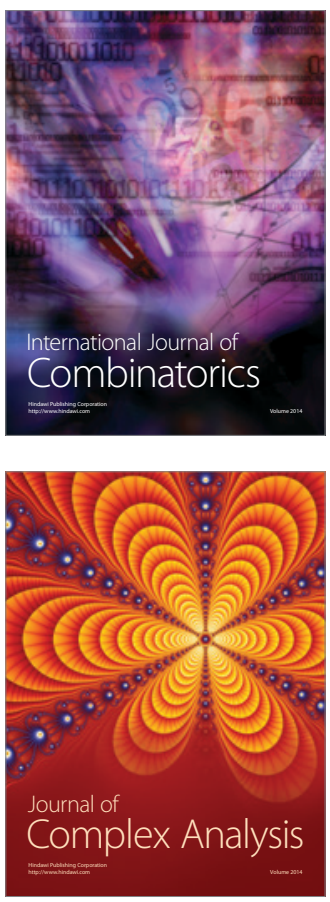

International Journal of

Mathematics and

Mathematical

Sciences
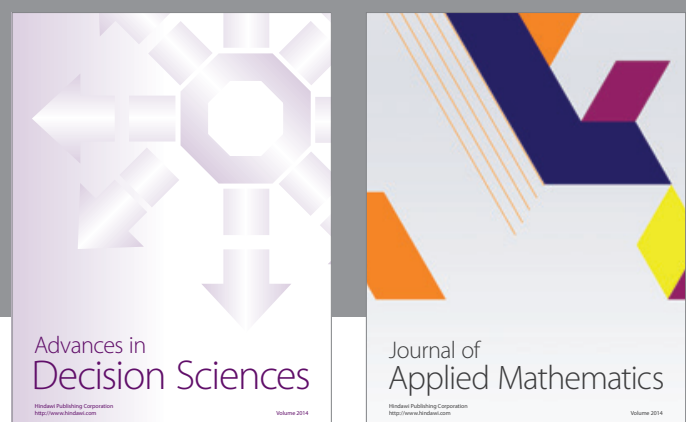

Journal of

Applied Mathematics
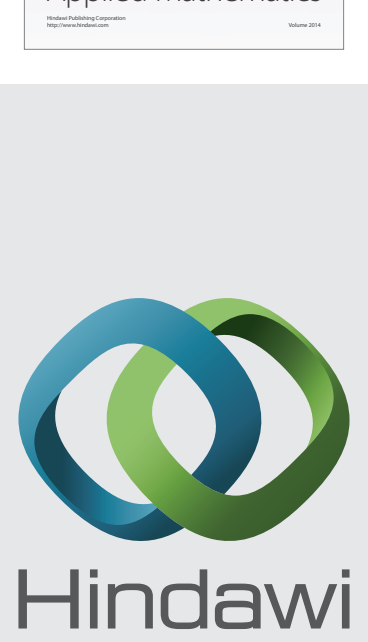

Submit your manuscripts at http://www.hindawi.com
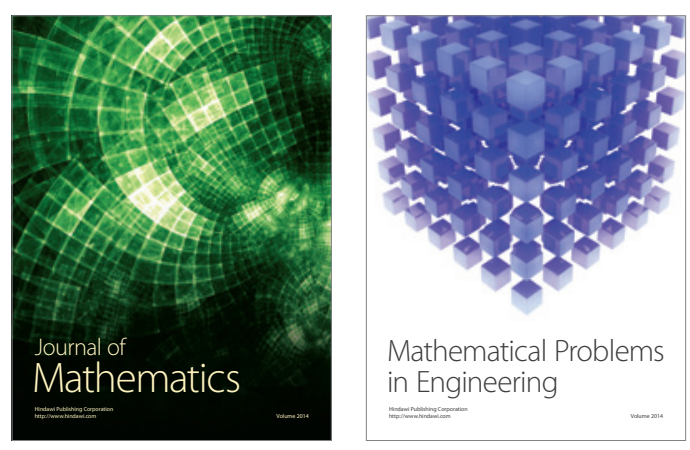

Mathematical Problems in Engineering
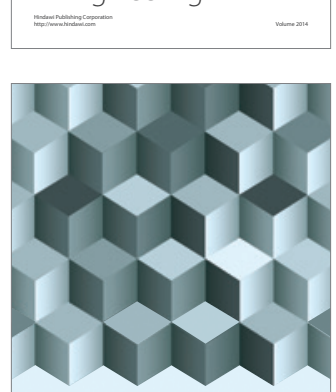

Journal of

Function Spaces
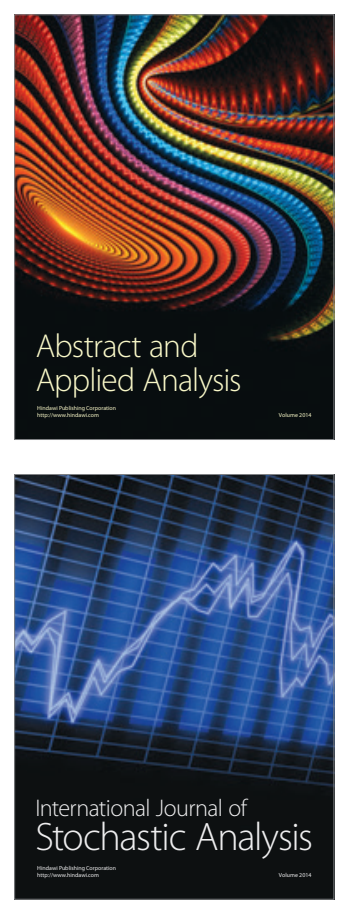

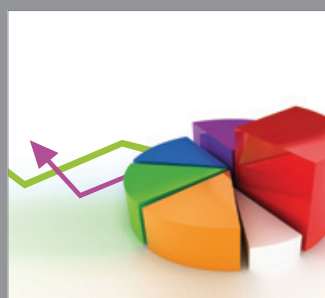

ournal of

Probability and Statistics

Promensencen
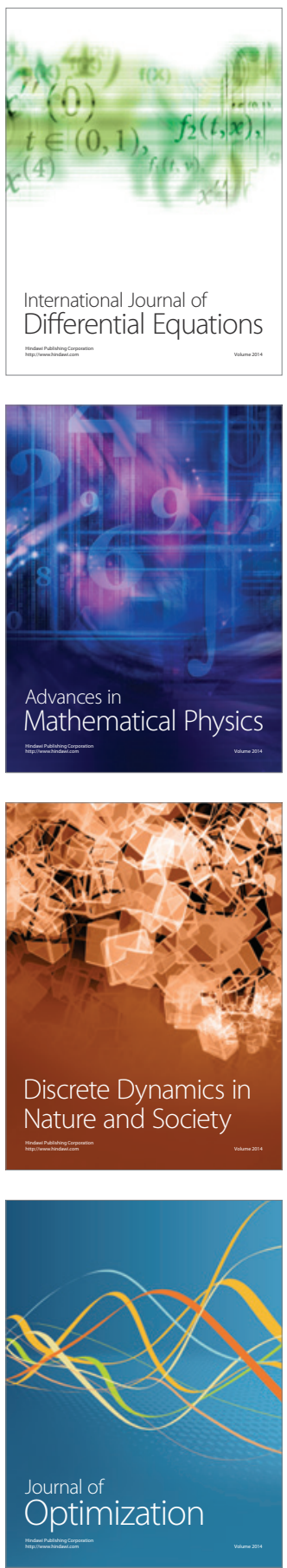